Image vignette

\section{Tarsal tunnel syndrome due to talocalcaneal coalition}

\author{
Chul Hyun Park ${ }^{1}$ (D), Mathieu Boudier-Revéret ${ }^{2}$ (D), \\ Min Cheol Chang ${ }^{3}$ (iD) \\ ${ }^{1}$ Department of Orthopedic Surgery, Yeungnam University College of \\ Medicine, Daegu, Korea \\ ${ }^{2}$ Department of Physical Medicine and Rehabilitation, Centre \\ hospitalier de l'Université de Montréal, Montréal, Canada \\ ${ }^{3}$ Department of Physical Medicine and Rehabilitation, Yeungnam \\ University College of Medicine, Daegu, Korea
}

Received: August 19, 2021 Revised: August 27, 2021

Accepted: August 28, 2021

Corresponding author: Min Cheol Chang, MD

Department of Physical Medicine and Rehabilitation, Yeungnam University

College of Medicine, 170 Hyeonchung-ro, Nam-gu, Daegu 42415, Korea

Tel: +82-53-620-4682 Fax: +82-53-4231-8694

E-mail:wheel633@gmail.com

J Yeungnam Med Sci 2023;40(1):106-108

https://doi.org/10.12701/yujm.2021.01473

Copyright ( $\odot 2023$ Yeungnam University College of Medicine, Yeungnam University Institute of Medical Science

This is an Open Access article distributed under the terms of the Creative Commons Attribution Non-Commercial License (http://creativecommons.org/ licenses/by-nc/4.0/) which permits unrestricted non-commercial use, distribution, and reproduction in any medium, provided the original work is properly cited.

A 26-year-old man visited the orthopedic department of our university hospital due to recently aggravated right medial ankle pain and tingling sensation over the plantar side of the right foot that had started 8 years ago without trauma. Symptoms were aggravated by walking on uneven surfaces. On physical examination, the range of motion of the subtalar joint was limited. Tinel sign was positive along the tibial nerve at the tarsal tunnel. Also, a double medial malleolus sign was observed, which represents the enlarged fused middle facet (Fig. 1A). On lateral ankle standing radiograph, the $\mathrm{C}$-sign, formed by the medial outline of the talar dome and posteroinferior aspect of the sustentaculum tali [1], was observed, and the bony prominence over the sustentaculum tali and medial process of talus was revealed (Fig. 1B). On computed tomography of the right ankle, talocalcaneal coalition was observed in the middle and posterior facets of the subtalar joint and involved about $40 \%$ of the joint on semicoronal image (Fig. 1C-1E). Magnetic resonance imaging showed the talocalcaneal coalition, and the tibial nerve was observed just adjacent to the talocalcaneal coalition, with the medial plantar nerve component compressed by it (Fig. $1 \mathrm{~F}-1 \mathrm{H})$. Coalition resection and subtalar joint arthrodesis were conducted. Intraoperatively, the tibial nerve was directly compressed and stretched over the talocalcaneal coalition (Fig. 1I). At 3-month follow-up, the patient's symptoms had nearly disappeared.

Tarsal tunnel syndrome (TTS) is a compressive neuropathy of the tibial nerve or one of its branches in the tarsal tunnel [2], which is a fibro-osseous channel located posterior to the medial malleolus of the ankle, and delineated by the talus, sustentaculum tali, medial calcaneal wall and the flexor retinaculum on the outside. Within the tarsal tunnel, the tibial nerve splits into three branches including calcaneal nerve (innervating the heel) and medial and lateral plantar nerves (innervating the bottom of the foot). TTS presents with paresthesias over the heel and plantar area of the foot [2]. Because the flexor retinaculum acts as a roof over an osseous tunnel, increment of pressure within the tunnel frequently causes compression of the nerves. There are several etiologies of TTS, including trauma, malalignment of the ankle joint, and space-occupying lesions such as ganglion or synovial cyst, and pigmented villonodular synovitis [3]. In our case, the talocalcaneal coalition increased the pressure on tibial nerve within tarsal tunnel causing TTS.

Talocalcaneal coalition is one of the common types of tarsal coalition. It is a fibrous, cartilaginous, or osseous union of $\geq 2$ of the tarsal bones [1] and develops because of disturbance or failure of mesenchymal segmentation [4]. In the general population, its incidence is reported to be approximately $1 \%$ to $6 \%$ [1]. Most symptomatic cases present during adolescence when the ossification of talocalcaneal bridge occurs.

If a patient complains of medial ankle pain with neurological symptoms on the plantar side of the foot, especially if symptoms began at adolescence, clinicians should consider the possibility of TTS due to talocalcaneal coalition. 

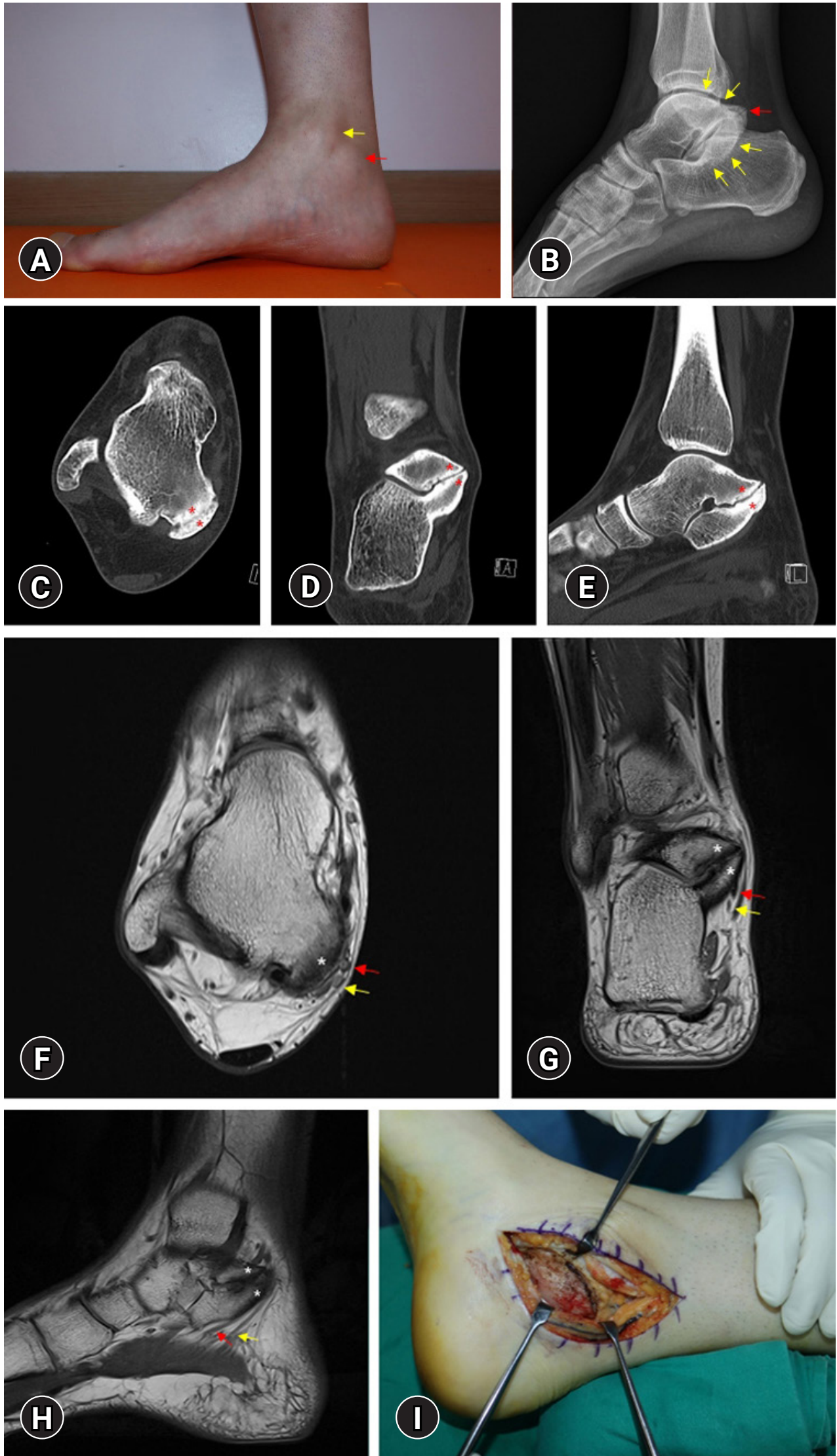

Fig. 1. (A) The prominence on the posterior inferior aspect of the right medial malleolus (red arrow,) called the "double medial malleolus sign" (yellow arrow, medial malleolus). (B) Lateral radiograph of the right ankle reveals a continuous C-shaped arc (yellow arrows), which is formed by the medial outline of the talar dome and posteroinferior aspect of the sustentaculum tali due to their bridging, and the bony prominence (red arrow) over the right sustentaculum tali and medial process of talus were observed. (C) Axial, (D) semicoronal, and (E) sagittal right ankle computed tomography show talocalcaneal coalition (asterisks) in the posterior subtalar joint. (F) Axial, $(G)$ coronal, and $(\mathrm{H})$ sagittal T2-weighted magnetic resonance imaging of the right ankle reveal the talocalcaneal coalition (asterisks) and tibial nerve adjacent to the coalition. The medial plantar nerve (red arrow) was compressed by the coalition (yellow arrows, lateral plantar nerve). (I) Intraoperative finding of talocalcaneal coalition. 


\section{Notes}

\section{Ethical statements}

This study was approved by the Institutional Review Board of the Yeungnam University Hospital (IRB No: 2021-06-061). Written informed consent was obtained for publication of this report and accompanying images.

\section{Conflicts of interest}

Chul Hyun Park and Mathieu Boudier-Revéret have been editorial board member of Journal of Yeungnam Medical Science (JYMS) since 2021. Min Cheol Chang has been Associate editor of JYMS since 2021. They were not involved in the review process of this manuscript. Otherwise, there is no conflict of interest to declare.

\section{Funding}

This study was supported by the National Research Foundation of Korea Grant funded by the Korean Government (No. NRF2021R1A2C1013073).

\section{Author contributions}

Conceptualization: all authors; Investigation, Data curation: CHP, MCC; Formal analysis, Funding acquisition, Supervision: MCC; Methodology: CHP; Visualization: MB, MCC; Writing-original draft: all authors; Writing-review \& editing: all authors.

\section{References}

1. Crim JR, Kjeldsberg KM. Radiographic diagnosis of tarsal coalition. AJR Am J Roentgenol 2004;182: 323-8.

2. Cimino WR. Tarsal tunnel syndrome: review of the literature. Foot Ankle 1990;11:47-52.

3. Mann RA. Disease of the nerve. In: Man RA, Coughlin MJ, editors. Surgery of the foot and ankle. St. Louis: Mosby; 1999. p. 512-6.

4. Guduri V, Dreyer MA. Talocalcaneal coalition [updated 2021 Mar 17]. In: StatPearls [Internet]. Treasure Island (FL): StatPearls Publishing; 2021 [cited 2021 Aug 5]. https://www.ncbi.nlm.nih.gov/books/ NBK549853/. 